\title{
ORTHOGONAL S-STEP METHODS FOR NONSYMMETRIC LINEAR SYSTEMS OF EQUATIONS
}

\author{
Charles D. Swanson \\ Cray Research, Inc., 655F Lone Oak Drive, Eagan, MN 55121 \\ Anthony T. Chronopoulos \\ Department of Computer Science, University of Minnesota, Minneapolis, MN 55455
}

\begin{abstract}
Conjugate Gradient-like methods such as Orthomin(k) have been developed to obtain a good numerical approximation to the solution of $A x=f$ when the matrix $A$ is large, sparse, and nonsymmetric. In s-step variations of these iterative methods, $s$ consecutive steps of the one-step methods are performed simultaneously. The number of inner products required is reduced and the resulting algorithms are more suitable for parallel computations. However, lack of orthogonality between the $s$ direction vectors at each iteration leads to instability unless $s$ is small $(s \leq 5)$. In this project, the effect of orthogonalizing the $s$ direction vectors at each iteration was studied. The $A^{T} A$-Orthogonal s-Step Orthomin(k) and pOrthogonal s-Step Orthomin(k) algorithms were developed and shown to be stable for large values of $s$ (up to $s=20$ ). The performance of these algorithms on a multiple processor CRAY Y-MP8 computer was analyzed.
\end{abstract}

1. Introduction. If $A$ is a nonsingular matrix of order $n$, an approximation to the solution of the linear system $A x=f$ can be obtained by the conjugate gradient (CG) method if $A$ is symmetric and positive definite. An approximate solution $x_{i}$ which minimizes the error functional

$$
E\left(x_{i}\right)=\left(x-x_{i}\right)^{T} A\left(x-x_{i}\right)
$$

is obtained at each iteration. The conjugate residual (CR) method is similar to $\mathrm{CG}$ but minimizes the residual

$$
E\left(x_{i}\right)=\left\|f-A x_{i}\right\|_{2}^{2} \text {. }
$$

In [1], Chronopoulos surveys variations of the CR method and develops s-step versions of the Generalized Conjugate Residual (GCR) and Orthomin(k) methods for nonsymmetric matrices with symmetric part $M=(A+$ $\left.A^{T}\right) / 2$ positive definite or indefinite. In s-step methods, $\mathrm{s}$ consecutive steps of the one-step methods are performed simultaneously.

Permission to copy without fee all or part of this material is granted provided that the copies are not made or distributed for direct commercial advantage, the $A C M$ copyright notice and the title of the publication and its date appear, and notice is given that copying is by permission of the Association for Computing Machinery. To copy otherwise, or to republish, requires a fee and/or specific permission. ICS'92-7/92/D.C.. USA

(c) 1992 ACM 0.89791-485-6/92/0007/0456 ..\$1.50
The generalized $C R$ method applied to nonsymmetric matrices minimizes $\left\|r_{1+1}\right\|_{2}^{2}$ along the direction $p_{i}$ in order to determine the steplength in $x_{i+1}=x_{i}+a_{i} p_{i} . p_{i}$ is made $A^{T} A$-orthogonal to $p_{i-1}$ but is not automatically $A^{T} A$ orthogonal to all of the previous $p_{j}$ 's as in the symmetric case; orthogonalization must be explicitly implemented. Positive definiteness assures that $a_{i}=\left(r_{i}, A p_{i}\right) /\left(A p_{l}, A p_{i}\right)$ is positive so the solution is improved at every step. Orthogonality and the norm reducing property of $C R$ guarantec convergence in at most $n$ iterations in the symmetric and positive definite case.

(i) Orthomin(k). For the GCR method, $p_{i}$ is $A^{T} A$ orthogonalized to all of the proceeding directions. In Orthomin $(\mathrm{k})$, orthogonalization is to only the preceding $k$ directions:

Algorithm 1.1. Orthomin(k)

1. $x_{0}, p_{0}=r_{0}=f-A x_{0}$

For $i=0$ Until Convergence Do

2. $\quad a_{1}=\frac{\left(r_{i}, A p_{i}\right)}{\left(A p_{i}, A p_{i}\right)}$

3. $x_{t+1}=x_{t}+a_{1} p_{t}$

4. $\quad r_{i+1}=r_{i}-a_{1} A p_{i}$

5. $\quad b_{j}^{i}=\frac{-\left(A r_{i+1}, A p_{1}\right)}{\left(A p_{1}, A p_{1}\right)}$

6. $\quad p_{i+1}=r_{i+1}+\sum_{j=i-k+1}^{i} b_{j}^{i} p$,

7. Compute $A p_{i+1}$

EndFor. 
(ii) s-step Orthomin(k). In the s-step Orthomin(k) method, the $s$ directions $\left\{r_{i}, \ldots, A^{s-1} r_{i}\right\}$ are formed and $A^{T} A$ orthogonalized to $k$ of the preceding directions $\left(p_{j}^{1}, \ldots, p_{j}^{s}\right\}$, $j=i-k+1, i$ The norm of the residual $\left\|r_{i+1}\right\|$ is minimized simultaneously in all $s$ new directions in order to obtain $x_{i+1}$. Following the detailed discussion in Section 4 of [1], let

$$
\begin{aligned}
& W_{i}=\left[\left(A p_{i}^{j}, A p_{i}^{i}\right)\right], \text { where } 1 \leq j, l \leq s \\
& \left.\underline{a}_{i}=\left[a_{i}^{1}, \cdots, a_{i}^{s}\right] \text { (the steplengths in updating } x_{i}\right) \\
& \underline{m}_{i}=\left[\left(r_{i}, A p_{i}^{1}\right), \cdots,\left(r_{i}, A p_{i}^{s}\right)\right]^{T} \\
& \underline{c}_{j}^{l}=\left[\left(A^{l} r_{i+1}, A p_{j}^{1}\right), \cdots,\left(A^{1} r_{i+1}, A p_{j}^{s}\right)\right]^{T} \\
& \underline{b}_{1}^{l}=\left\{b_{,}^{(l, m)}\right\}_{m=1}^{s} \text { for } j=i-k+1, \cdots, i \text { and } l=1, \cdots, s
\end{aligned}
$$

(the coefficients to $A^{T} A$ - orthogonalize to the previous directions)

$$
\begin{aligned}
& P_{1}=\left[p_{1}^{1}, \cdots, p_{i}^{s}\right] \text { (the direction vectors) } \\
& R_{i}=\left[r_{1}, A r_{i}, \cdots, A^{s-1} r_{i}\right] \text { (the residuals) }
\end{aligned}
$$

Then a description of the s-step Orthomin(k) method can be given as follows:

\section{Algorithm 1.2. s - step Orthomin(k)}

1. $x_{0}, P=\left[r_{0}=f-A x_{0}, A r_{0}, \cdots, A^{s-1} r_{0}\right]$

\section{For $i=0$ Until Convergence Do}

$$
\text { 2. Compute } \underline{\mathrm{m}}_{i}, W_{1}
$$

3. Call Scalar1

4. $x_{i+1}=x_{i}+P_{i} \underline{a}$.

5. $\quad r_{i+1}=r_{i}-A P_{i} \underline{a}_{i}$

6. Compute $\underline{c}_{s}^{i}, \quad j=i-k+1, \cdots, i$

7. Call Scalar2

8. Compute $R_{2}=\left[r_{i}, A r_{i}, \cdots, A^{s-1} r_{i}\right]$

9. $P_{i+1}=R_{i+1}+\sum_{j=i-k+1}^{\dot{1}} P_{j}\left[\underline{b}_{j}^{l}\right]_{i=1}^{s}$
10. Compute $A P_{i+1}$ or,

11. $A P_{i+1}=A R_{i+1}+\sum_{j=i-k+1}^{1} A P,\left[\underline{b}^{l}\right]_{i=1}^{s}$

\section{EndFor.}

Scalarl: Decomposes $W_{i}=\left[\left(A p_{i}^{\prime}, A p_{i}^{\prime}\right)\right]$, where $1 \leq j, l \leq s$. It then solves $W_{\imath} \underline{a}_{2}=\underline{m}_{\imath}$, where $\underline{a}_{\imath}=\left[a_{1}^{1}, \cdots, a_{i}^{s}\right]^{T}$ and $\underline{m}_{i}=\left[\left(r_{1}, A p_{1}^{1}\right), \cdots,\left(r_{1}, A p_{\mathrm{a}}^{s}\right)\right]^{T}$.

Scalar2: Solves $W \underline{b}^{2}=-\underline{c}^{2}$, for $j=i-k+1, \cdots, i$ and $l=1, \cdots, s$ where $\underline{c}^{l}=\left[\left(A^{i} r_{i+1}, A p^{1}\right), \cdots,\left(A^{l} r_{i+1}, A p_{j}^{s}\right)\right]^{T}$ and $\underline{b}_{j}^{l}=\left\{b_{,}^{(l, m)}\right\}_{m=1}^{s}$ for $j=1-k+1, \cdots, i$ and $l=1, \cdots, s$.

The solution of the Scalar1 and Scalar2 linear systems may cause a quick loss of orthogonality of the $s$-dimensional direction subspaces $P_{i}$. Non-orthogonality of the $s$ vectors can lead to instability in the algorithm as $s$ gets larger than $5[1,3]$. The purpose of this project is to determine if orthogonalizing the $s$ direction vectors within each subspace $P_{i}$ can lead to a stable algorithm for larger values of $s$ thereby reducing the number of iterations required. The increased work in each iteration can be executed in parallel.

(iii) Numerical Experiments. The numerical experiments carried out utilized two test problems. The first problem was derived from a five point discretization of the partial differential equation

$$
-\left(b u_{x}\right)_{x}-\left(c u_{x}\right)_{x}+d u_{x}+\left(d u_{x}\right)_{x}+e u_{y}+\left(e u_{y}\right)_{y}+f u=g
$$

on the unit square, where

$$
\begin{aligned}
& b(x, y)=e^{-x y}, c(x, y)=e^{x y}, d(x, y)=b(x+y) \\
& e(x, y)=(x+y) \text { and } f(x, y)=1 . /(1+x+y)
\end{aligned}
$$

subject to Dirichlet boundary conditions $u=0$. The right hand side $g$ was chosen so that the solution was known to be $e^{x y} \sin (\pi x) \sin (\pi x)$. The parameters were chosen to produce a nonsymmetric matrix. This problem will be referred to as the "PDE problem."

The second test problem is from Walker [4] and is used to evaluate the numerical stability of the various algorithms. 
The matrix $A$ is

$$
A=\left[\begin{array}{ccccccc}
1 & 0 & . & . & . & 0 & \alpha \\
0 & 2 & . & . & . & \cdot & 0 \\
\cdot & \cdot & . & & & . & . \\
\cdot & . & & . & & . & . \\
. & . & & & . & \cdot & . \\
0 & . & . & . & . & n-1 & 0 \\
0 & 0 & . & . & . & 0 & n
\end{array}\right]
$$

while the right hand side $b=(1, \ldots, 1)^{T}$. The problem was solved for $n=100$ with $a=2 \times 10^{6}$. This problem will be referred to as the "ill-conditioned problem." The matrix condition number (in the infinity norm) is greater or equal to $\alpha^{2}$.

2. $A^{T} A$-Orthogonal $\mathrm{s}$-Step Orthomin(k). In this method the direction vectors within each subspace $P_{i}$ are $A^{T} A$-orthogonalized using the Modified Gram-Schmidt method. The Scalar1 and Scalar2 linear systems in Algorithm 1.2 need not be solved in the $A^{T} A$-Orthogonal sStep Orthomin(k) method since the $W_{i}$ matrix is the identity matrix if $P_{i}$ is perfectly $A^{T} A$-orthogonalized. The algorithm is as follows:

Algorithm 2.1. $A^{\mathrm{T}} \mathrm{A}$ - Orthogonal s - step Orthomin(k)

1. $x_{0}, P=\left[r_{0}=f-A x_{0}, A r_{0}, \cdots, A^{s-1} r_{0}\right]$

For $\mathrm{i}=0$ Until Convergence Do

2. Compute $\underline{\mathrm{a}}_{i}$

3. $x_{i+1}=x_{i}+P_{i} \underline{a}_{i}$

4. $\quad r_{i+1}=r_{1}-A P_{i} a_{i}$

5. Compute $\underline{b}^{t}$

6. Compute $R_{t}=\left[r_{i}, A r_{i}, \cdots, A^{s-1} r_{i}\right]$

7. $P_{i+1}=R_{i+1}+\sum_{j=i-k+1}^{i} P_{j}\left[\underline{b}_{j}^{i}\right]_{i=1}^{s}$

8. Compute $A P_{i+1}$ or,

9. $A P_{i+1}=A R_{i+1}+\sum_{j=i-k+1}^{i} A P_{i}\left[\underline{b}_{i}^{i}\right]_{i=1}^{s}$
10. $A^{T} A$-orthogonalize $A P_{i+1}$ using the Modified

Gram - Schmidt method to obtain $\underline{A P}_{i+1}$ and $\underline{P}_{i+1}$

\section{EndFor.}

Here $\underline{A P}_{i+1}$ is orthogonalized.

Results for the PDE problem are shown in Table 2.1 for the s-Step method and the $A^{T} A$-Orthogonal s-Step method. The initial values $x(i)=0.05^{*} \bmod (i, 50)$ were used. The stopping criterion was $\left\|r_{i}\right\|_{2} \leq 10^{-6}$ with the maximun number of iterations allowed set to 700 . The number of iterations to convergence, maximum error, and CPU time are listed. The maximum error is the maximum value of $a b s[x(i)-s o l(i)]$ where $x(i)$ is the calculated value and sol(i) is the known solution. The $s=1$ case is standard Orthomin(k). For $\mathrm{k}=1$ and $\mathrm{s}>1$, the maximum error for the $\mathrm{s}$-Step method shows a dramatic increase at $s=12$ while the Orthogonal $s-$ step method maintains the initial maximum error through $s=16$. At $s=20$ the error increases very quickly. For $k=2$ and $k=4$, the $s-S t e p$ method fails at $s=12$. The Orthogonal method is stable through $\mathrm{s}=16$ for $\mathrm{k}=2$ and again shows an increase in the maximum error at $s=20$. For $k=4$, the Orthogonal method shows an increase in the number of iterations at $s=16$ and an increase in the maximum error at $s=20$.

To test the effect of removing the $s \times s$ linear system solvers, a set of runs was made with $A^{T} A$-orthogonalization of $P_{l}$, but with the linear systems in place. The results in Table 2.1 show minimal effect for the PDE problem.

Results for the ill-conditioned problem which severely tests the numerical stability of the algorithms are shown in Table 2.2. The advantage of the Orthogonal s-Step method versus the $s$-Step method is apparent at $s=8, k=1$ where the iteration count and maximum error for the $s$-Step method begin to increase. The Orthogonal method remains stable through $s=12$. For tests with $k=4$, the iteration count is twice as high for the s-Step method at $s=4$.

Again, runs with the linear system solvers in place were made for the ill-conditioned problem. There were some improvements at $\mathrm{s}=16$ for both $\mathrm{k}=1$ and $\mathrm{k}=4$ but the maximum error was still high (Table 2.2).

To test the effect of the stopping criterion, the parameter "eps" was tightened from $10^{-10}$ to $10^{-12}$. The results are shown in Table 2.3. At $\mathrm{s}=8$, the maximum error for the Orthogonal method has improved by a factor of 65 but at $s=12$ it is roughly the same for the two cases. 
3. p-Orthogonal s-Step Orthomin(k). An alternative to $A^{T} A$-orthogonalizing the direction vectors is to simply orthogonalize the direction vectors within the subspace $P_{i}$ directly. This is simple to implement, but does not diagonalize the matrices in the linear systems of Algorithm 1.2 so they still have to be solved. This is called the p-Orthogonal s-step Orthomin(k) algorithm:

Algorithm 3.1. p - Orthogonal s - step Orthomin(k)

1. $x_{0}, P=\left[r_{0}=f-A x_{0}, A r_{0}, \cdots, A^{s-1} r_{0}\right]$

For $i=0$ Until Convergence Do

2. Compute $\underline{\mathrm{m}}_{i}, W_{i}$

3. Call Scalar1

4. $x_{i+1}=x_{i}+P_{i} \underline{a}_{i}$

5. $r_{i+1}=r_{i}-A P_{i} \underline{a}_{i}$

6. Compute $\underline{c}_{j}^{i}, \quad j=i-k+1, \cdots, i$

7. Call Scalar2

8. Compute $R_{i}=\left[r_{i}, A r_{i}, \cdots, A^{s-1} r_{i}\right]$

9. $\quad P_{i+1}=R_{i+l}+\sum_{j=i-k+1}^{i} P_{j}\left[\underline{b}_{j}^{l}\right]_{l=1}^{s}$

10. Orthogonalize $P_{i+1}$ using a Modified

Gram - Schmidt or Householder

method to obtain $\underline{P}_{i+1}$

11. Compute $A \underline{P}_{i+1}$

EndFor.

Orthogonalization of the direction vectors was done with the Modified Gram-Schmidt method and also with the Householder technique. The Modified Gram-Schmidt is generally more efficient but the Householder technique is more accurate for ill-conditioned matrices [5, p. 219]. However, the Householder method is also more expensive.

Table 3.1 shows results for the PDE problem using s-Step Orthomin(k) and p-Orthogonal s-Step Orthomin(k) with Modified Gram-Schmidt and Householder orthogonalization. The s-Step algorithm begins to show instability at $\mathrm{s}=12$ (for $\mathrm{k}=1$ ). The Orthogonal algorithm is stable through $s=20$, with the maximum error having the same order of magnitude throughout for both Modified Gram-Schmidt and Householder orthogonalization.

For $\mathrm{k}>1$, the $\mathrm{p}$-Orthogonal s-Step method shows a higher iteration count than the s-Step method at $s=4$, especially for $\mathrm{k}=4$. The direction vectors in $P_{i}$ which are $A^{T} A$ orthogonalized to the $\mathrm{k}$ previous dirction vectors in the other orthomin(k) methods discussed have been $\mathrm{p}$ orthogonalized here. This lack of $A^{T} A$-orthogonalization to the previous $\mathrm{k}$ vectors means that the $\mathrm{p}$-Orthogonal s-Step Orthomin(k) algorithm is not effective for $k>1$ (see illconditioned problem results at $\mathrm{k}=2$ in Table 3.2).

Table 3.2 compares both orthogonalization methods applied to the ill-conditioned problem with $n=100$ and $\alpha=2 \times 10^{6}$ and eps $=1 \times 10^{-10}$. For $\mathrm{k}=1$ both orthogonal methods are stable through $s=20$ (the $s$-Step method failed at $s=8$ ). However, for large values of $s(16$ and 20$)$ the Householder orthogonalization produced maximum errors an order of magnitude smaller than the Modified Gram-Schmidt method which can be expected.

For $\mathrm{k}=4$, the $\mathrm{p}$-Orthogonal method shows a much higher iteration count at $\mathrm{k}=2$, due to the lack of $A^{T} A$ orthogonalization to the previous $\mathrm{k}$ vectors.

4. Performance. Numerical experiments were executed on a CRAY Y-MP8 at Cray Research, Inc. in Eagan, MN. This computer has eight processors, a 6 nanosecond clock period, and 128 million 64-bit words of shared memory. Performance analysis tools available with the UNICOS 6.0 operating system were used.

\section{(i) Single Processor Performance.}

The PERFTRACE performance tool uses the hardware performance monitors on the CRAY Y-MP to determine the amount of vectorization in the code and measure the performance in Megaflops for each routine. The statistics in Table 4.1 for the $A^{T} A$-Orthogonal s-Step Orthomin(k) code indicate that it is highly vectorized, since the peak performance on a single CRAY Y-MP processor is 330 Megaflops.

The CPU seconds for the PDE problem with $\mathrm{N}=130$ (where $\mathrm{N}^{2}$ is the order of matrix $A$ ) are included in Tables 2.1 and 3.1. The standard Orthomin(k) method is obtained by setting $s=1$ in the $s$-Step code. The best single processor timing for Orthomin $(k)$ was obtained for $k=2$. The best $\mathrm{s}$-Step Orthomin(k) timing was obtained with $\mathrm{s}=2$ and $k=1$. For both Orthogonal s-Stcp algorithms the best single processor timings were also obtained with $s=2$ and $k=1$. These four codes were then executed for problems 
with $\mathrm{N}$ ranging from 32 to 256 with the results shown in Table 4.2.

The Orthogonal methods take more time than the s-Step method because of the added work to orthogonalize the $P_{i}$ direction vectors. Since the linear systems are not solved in the $A^{T} A$-Orthogonal method, it is faster than the $\mathrm{p}$ Orthogonal method.

\section{(ii) Multiple Processor Performance}

The codes were executed in parallel on the CRAY Y-MP using the Autotasking feature of the Fortran compiling system which provides automatic multitasking over the eight processors. The parallel processing performance was studied and measured using the "Atexpert" tool, an expert system that predicts the expected performance on a dedicated system from runs made on a nondedicated system. The programmer can use the information provided by Atexpert along with compiler directives explicitly inserted in the code to iteratively enhance the parallel performance of the program.

For the $A^{T} A$-Orthogonal method, Atexpert showed that the initial version of the program was $60 \%$ parallel. Amdahl's Law gives a maximum speedup of 1.4 on an 8 processor system for this level of parallelism. The Atexpert tool helps the programmer identify the parallel and serial regions in the program. Once the troublesome serial regions have been identified, compiler directives can then be inserted to provide enhanced parallel execution. A simple example is to tell the compiler to execute an inner loop on multiple processors, when ordinarily it would not. Since the inner loops for the largest problem are 65,536 elements long $(\mathrm{N}=256)$ this was effective. Directives can also be inserted to tell the compiler when potential dependencies inhibiting parallel code generation can safely be ignored. Using these techniques, the serial regions of the $A^{T} A$-Orthogonal code were attacked in order to provide a highly parallel program.

The value of orthogonalizing the $P_{i}$ direction vectors is that it allows $s$ to become larger. This means the number of iterations is reduced with more work being done in each iteration, a situation that should make more efficient use of multiple processors. The additional work from the orthogonalization should be compensated by the enhanced parallel performance to obtain a faster algorithm.

Multiple processor runs were made on a dedicated CRAY YMP8 for the Orthomin(k), s-step Orthomin(k), and $A^{T} A$ Orthogonal s-step Orthomin(k) algorithms, using the large $\mathrm{N}=256$ PDE problem. The results are given in Table 4.3 and illustrated in Figure 4.1. Only $s=2$ and $s=4$ results are shown for the s-Step method since the numerical accuracy tests with this method show loss of accuracy at $\mathrm{s}=8$ (Table
3.2). For the $A^{T} A$-Orthogonal method, $s$ values of 2,4 , and 8 are shown $(\mathrm{k}=1)$. The best absolute performance for this problem was achieved with the $A^{T} A$-Orthogonal 4-step Orthomin(1) method using eight processors. However, the speed-up factor of 3.76 on an eight processor system indicates that maximum parallel performance is not being achieved. This is due to remnants of serial code plus overhead. A significant source of overhead (identified with help from the Atexpert tool) is "load imbalance" in parallelizing the Modified Gram-Schmidt orthogonalization routine.

Using two processors, the load imbalance problem is largely alleviated and the parallel $A^{T} A$-Orthogonal method is efficient, as shown by the 1.85 speed-up. Nevertheless, for the two processor runs the elapsed time for the 2-step Orthomin(1) method was significantly better. Therefore, unless the user has a dedicated eight processor machine, 2step Orthomin(1) is the most effective algorithm.

5. Summary. This project demonstrated that the value of $s$ in the s-Step Orthomin $(\mathrm{k})$ algorithm can be increased beyond $s=5$ by orthogonalizing the $s$ direction vectors in each iteration. Two orthogonalization schemes were presented. The $A^{T} A$-Orthogonal s-Step Orthomin(k) algorithm provided good numerical accuracy through $s=12$ $(\mathrm{k}=1)$ for the ill-conditioned problem. For $\mathrm{k}>1$, the $A^{T} A$ Orthogonal method was stable through $s=8$. The $p$ Orthogonal s-Step Orthomin $(k)$ showed stability through $s=20 \quad(k=1)$ with the Householder orthogonalization showing significantly greater numerical accuracy than Modified Gram-Schmidt at $s=16$ and $s=20$. However, the $p-$ Orthogonal method does not work well for $k>1$. For the PDE problem, both methods were stable through $s=20$ $(k=1)$.

Performance measurements of the algorithms on a CRAY Y-MP8 computer showed the $A^{T} A$-Orthogonal 4-step Orthomin(1) algorithm to be fastest using all eight processors. However, the 2-step Orthomin(1) algorithm using two processors was only $7 \%$ slower. Improvements in the parallel performance of the Orthogonal s-Step algorithms followed by implementation on systems with more than eight processors (such as the sixteen processor CRAY Y-MP C90) may show the Orthogonal s-Step algorithms to be superior to the s-Step method.

Also note that in practice, preconditioning is used to improve the condition number of the coefficient matrix and speed up the convergence of the linear system.

6. Acknowledgements. This research was partially supported by supported by NSF grant CCR-8722260. The Minnesota Supercomputer Institute provided time on a CRAY-2. CRAY Y-MP time was provided by Cray 
Research, Inc. We wish to thank the reviewers for comments that enhanced the quality of the presentation.

\section{References}

[1] A. T. Chronopoulos, "s-Step Iterative Methods for (Non)symmetric (In)definite Linear Systems," SIAM J. of Numerical Analysis, Vol. 28, No. 6, Dec. 1991.

[2] A. T. Chronopoulos and C. W. Gear, "s-Step Iterative Methods for Symmetric Linear Systems," $J$. of Computational and Applied Math., Vol. 25, 1989, p. 153-168.
[3] Anthony T. Chronopoulos, "s-Step Orthomin and GMRES Implemented on Parallel Computers," Tech. Rep. CSci No. TR 90-15, U. of Minnesota, February 1990.

[4] H. Walker, "Implementation of the GMRES Method Using Householder Transformations," SIAM J. SCi. Stat. Comp., 9 (1988), pp. 152-163.

[5] Gene H. Golub and Charles F. Van Loan, Matrix Computations, second edition, Johns Hopkins, 1989.

Table 2.1. $A^{T} A-$ Orthogonal s-Step Orthomin(k) Compared to s-Step Ortomin(k) for PDE Problem.

\begin{tabular}{|c|c|c|c|c|c|c|c|c|c|c|}
\hline \multirow[b]{2}{*}{ s } & \multirow[b]{2}{*}{ k } & \multicolumn{3}{|c|}{ s-Step Orthomin } & \multicolumn{3}{|c|}{$\begin{array}{c}A^{T} A \text {-Orthogonal s-Step Orthomin } \\
\text { Modified Gram-Schmidt } \\
\text { No Linear Systems }\end{array}$} & \multicolumn{3}{|c|}{$\begin{array}{c}A^{T} A \text {-Orthogonal s-Step Orthomin } \\
\text { Modified Gram-Schmidt } \\
\text { With Linear Systems }\end{array}$} \\
\hline & & iterations & max error & CPU time & iterations & max error & CPU time & iterations & max error & CPU time \\
\hline 1 & 1 & 471 & 0.0003959 & 0.942 & - & - & - & - & - & - \\
\hline 2 & 1 & 173 & 0.000558 & 0.824 & 173 & 0.0005563 & 1.116 & 173 & 0.0005561 & 1.187 \\
\hline 4 & 1 & 88 & 0.0003412 & 1.129 & 88 & 0.0003414 & 1.485 & 88 & 0.0003414 & 1.6 \\
\hline 8 & 1 & 63 & 0.0004254 & 2.444 & 63 & 0.0003623 & 3.167 & 64 & 0.0004789 & 3.532 \\
\hline 12 & 1 & 48 & 0.0191 & 3.738 & 44 & 0.0003969 & 4.426 & 44 & 0.0003975 & 4.884 \\
\hline 16 & 1 & 49 & 0.0568 & 6.357 & 31 & 0.0003831 & 5.164 & 30 & 0.0002646 & 5.56 \\
\hline 20 & 1 & 55 & 0.0952 & 10.843 & 28 & 0.005214 & 6.924 & 26 & 0.00552 & 7.219 \\
\hline 1 & 2 & 368 & 0.0003892 & 0.874 & - & - & - & - & - & - \\
\hline 2 & 2 & 179 & 0.0003959 & 1.104 & 179 & 0.0003957 & 1.411 & 179 & 0.0003959 & 1.476 \\
\hline 4 & 2 & 108 & 0.0002909 & 1.976 & 108 & 0.000373 & 2.436 & 109 & 0.0002356 & 2.583 \\
\hline 8 & 2 & 66 & 0.0002608 & 3.97 & 78 & 0.0004748 & 5.68 & 72 & 0.0005102 & 5.561 \\
\hline 12 & 2 & 69 & 0.07796 & 8.76 & 51 & 0.0004469 & 7.639 & 49 & 0.0003899 & 7.835 \\
\hline 16 & 2 & $* * *$ & $* * *$ & $* * *$ & 37 & 0.000329 & 9.382 & 36 & 0.0002384 & 9.69 \\
\hline 20 & 2 & $* * *$ & $* * *$ & $* * *$ & 29 & 0.01133 & 11.146 & 30 & 0.000411 & 12.183 \\
\hline 1 & 4 & 353 & 0.000404 & 1.091 & - & - & - & - & - & - \\
\hline 2 & 4 & 213 & 0.0004432 & 1.91 & 213 & 0.0004332 & 2.345 & 213 & 0.0004253 & 2.358 \\
\hline 4 & 4 & 125 & 0.000379 & 3.708 & 129 & 0.000281 & 4.419 & 130 & 0.0003655 & 4.467 \\
\hline 8 & 4 & 148 & 0.000283 & 15.519 & 78 & 0.0002489 & 9.15 & 80 & 0.0003099 & 9.557 \\
\hline 12 & 4 & $* * *$ & $* * *$ & $* * *$ & 54 & 0.000275 & 13.131 & 52 & 0.0003671 & 13.227 \\
\hline 16 & 4 & $* * *$ & $* * *$ & $* * *$ & 60 & 0.0001806 & 25.198 & 60 & 0.0001419 & 26.516 \\
\hline \multirow[t]{2}{*}{20} & 4 & $* * *$ & $* * *$ & $* * *$ & 54 & 0.0193 & 34.575 & 55 & 0.0252 & 37.447 \\
\hline & & $\begin{array}{l}\text { CPU tir } \\
s=1 \text { is } s\end{array}$ & $\begin{array}{l}\mathrm{e}=1 \text { proce } \\
\text { andard Orthe }\end{array}$ & $\begin{array}{l}\text { sor CPU tin } \\
\operatorname{nin}(k)\end{array}$ & in second & & \multicolumn{4}{|c|}{ eps $=1.0 \mathrm{E}-6 \quad \mathrm{~N}=130$} \\
\hline
\end{tabular}


Table 2.2. $A^{T} A$-Orthogonal s-Step Orthomin Compared to s-Step Orthomin for Ill-Conditioned Problem (eps=1.0E-10).

\begin{tabular}{|c|c|c|c|c|c|c|c|}
\hline \multirow[b]{2}{*}{$\mathrm{s}$} & \multirow[b]{2}{*}{$\mathrm{k}$} & \multicolumn{2}{|c|}{ s-Step Orthomin } & \multicolumn{2}{|c|}{$\begin{array}{c}A^{T} A \text {-Orthogonal s-Step Orthomin } \\
\text { Modified Gram-Schmidt } \\
\text { No Linear Systems }\end{array}$} & \multicolumn{2}{|c|}{$\begin{array}{l}A^{T} A-\text { Orthogonal s-Step Orthomin } \\
\text { Modified Gram-Schmidt } \\
\text { With Linear Systems }\end{array}$} \\
\hline & & iterations & $\max$ error & iterations & $\max$ error & iterations & $\max$ error \\
\hline 1 & 1 & $701^{*}$ & 18811 & - & - & - & - \\
\hline 2 & 1 & 44 & 0.0001447 & 44 & 0.0003767 & 44 & 0.0003768 \\
\hline 4 & 1 & 29 & 0.0006337 & 23 & 0.0001111 & 23 & 0.0001111 \\
\hline 8 & 1 & 21 & 0.00207 & 10 & 0.0004373 & 10 & 0.000437 \\
\hline 12 & 1 & 22 & 0.0175 & 7 & 0.0003538 & 7 & 0.0003266 \\
\hline 16 & 1 & 30 & 41.267 & 13 & 83.833 & 6 & 0.4465 \\
\hline 1 & 4 & $701^{*}$ & 14556 & - & - & - & - \\
\hline 2 & 4 & 38 & 0.0001134 & 38 & 0.0001409 & 38 & 0.0001404 \\
\hline 4 & 4 & 37 & 0.0006885 & 18 & 0.0004195 & 18 & 0.0004215 \\
\hline 8 & 4 & 94 & 305.87 & 28 & 0.00002342 & 28 & 0.0001475 \\
\hline 12 & 4 & 102 & 5.036 & 41 & 0.001952 & 36 & 0.0006365 \\
\hline 16 & 4 & $* * *$ & $* * *$ & 76 & 205.99 & 46 & 3.99 \\
\hline \multicolumn{4}{|c|}{$\begin{array}{l}\text { NOTE: alpha }=2.0 \mathrm{E}+6 \\
\mathrm{~s}=1 \text { is standard Orthomin }(\mathrm{k})\end{array}$} & \multicolumn{4}{|c|}{$*=$ problem reached iteration count limit } \\
\hline
\end{tabular}

Table 2.3. $A^{T A} A$-Orthogonal s-Step Orthomin Compared to s-Step Orthomin for Ill-Conditioned Problem (eps=1.0E-12).

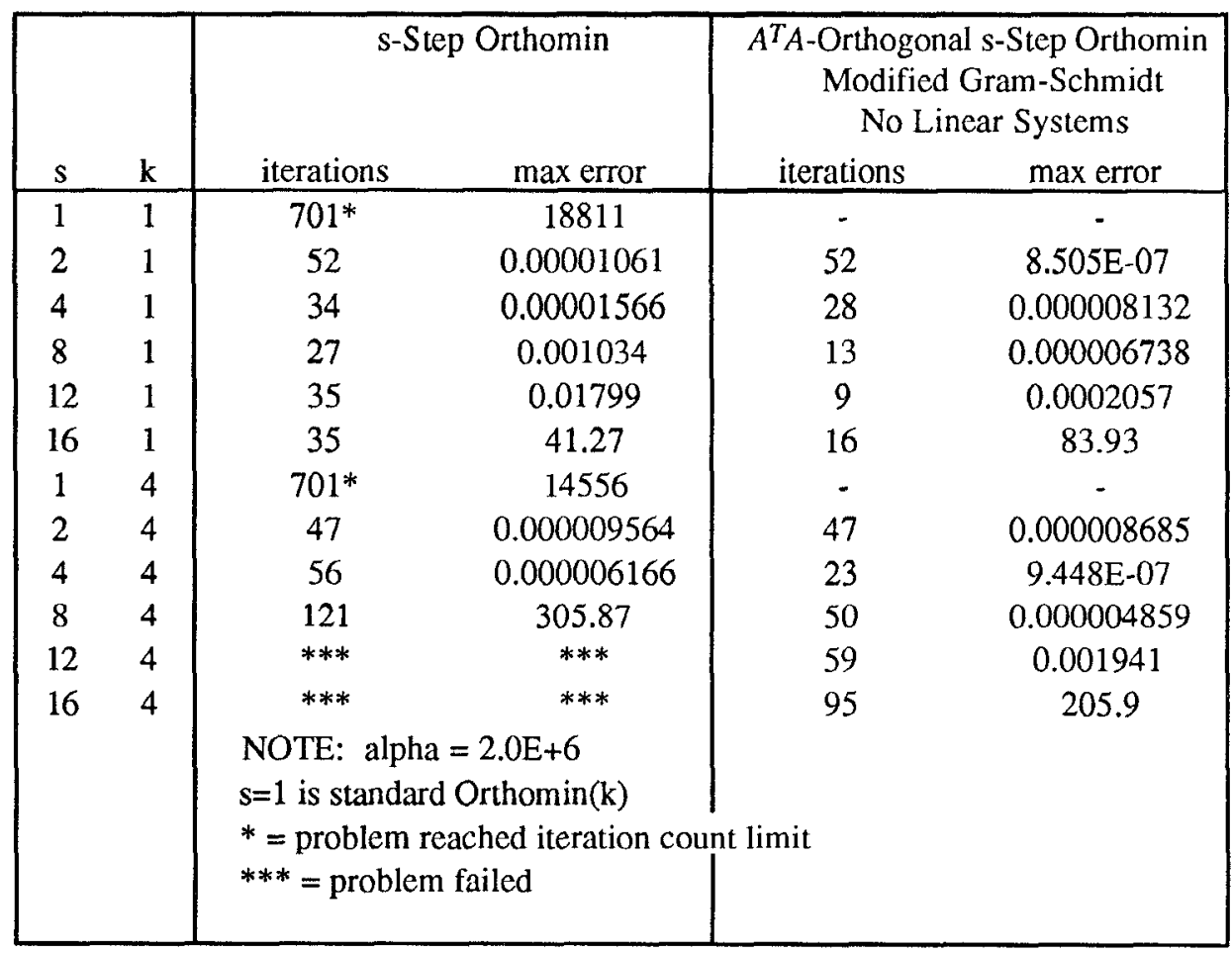


Table 3.1. p-Orthogonal s-Step Orthomin Compared to s-Step Orthomin for PDE Problem.

\begin{tabular}{|c|c|c|c|c|c|c|c|c|c|c|}
\hline \multirow[b]{2}{*}{$\mathrm{s}$} & \multirow[b]{2}{*}{$\mathrm{k}$} & \multicolumn{3}{|c|}{ s-Step Orthomin } & \multicolumn{3}{|c|}{$\begin{array}{l}\text { p-Orthogonal s-Step Orthomin } \\
\text { Modified Gram-Schmidt }\end{array}$} & \multicolumn{3}{|c|}{$\begin{array}{l}\text { p-Orthogonal s-Step Orthomin } \\
\text { Householder }\end{array}$} \\
\hline & & iterations & max error & CPU time & iterations & max error & CPU time & iterations & $\max$ error & CPU time \\
\hline 1 & 1 & 471 & 0.0003959 & 0.942 & - & - & - & - & - & - \\
\hline 2 & 1 & 173 & 0.000558 & 0.824 & 173 & 0.0006081 & 1.278 & 173 & 0.0006058 & 1.791 \\
\hline 4 & 1 & 88 & 0.0003412 & 1.129 & 88 & 0.0003415 & 1.662 & 88 & 0.0003419 & 2.368 \\
\hline 8 & 1 & 63 & 0.0004254 & 2.444 & 61 & 0.0004992 & 3.374 & 63 & 0.0004001 & 4.972 \\
\hline 12 & 1 & 48 & 0.0191 & 3.738 & 44 & 0.0004287 & 4.788 & 43 & 0.0003613 & 6.723 \\
\hline 16 & 1 & 49 & 0.0568 & 6.357 & 32 & 0.0004153 & 5.733 & 33 & 0.0003282 & 8.525 \\
\hline 20 & 1 & 55 & 0.0952 & 10.843 & 26 & 0.0002288 & 6.88 & 26 & 0.0003472 & 10.003 \\
\hline 1 & 2 & 368 & 0.0003892 & 0.874 & - & - & - & & & \\
\hline 2 & 2 & 179 & 0.0003959 & 1.104 & 179 & 0.0003958 & 1.547 & 179 & 0.0003959 & 2.058 \\
\hline 4 & 2 & 108 & 0.0002909 & 1.976 & 118 & 0.0004998 & 2.813 & 110 & 0.0003456 & 3.48 \\
\hline 8 & 2 & 66 & 0.0002608 & 3.97 & 71 & 0.0005862 & 5.285 & 78 & 0.000562 & 7.632 \\
\hline 12 & 2 & 69 & 0.07796 & 8.76 & 48 & 0.0003256 & 7.256 & 57 & 0.0003229 & 11.307 \\
\hline 16 & 2 & $* * *$ & $* * *$ & $* * *$ & 37 & 0.0002498 & 9.4 & 57 & 0.0005329 & 19.053 \\
\hline 1 & 4 & 353 & 0.000404 & 1.091 & - & - & - & - & - & - \\
\hline 2 & 4 & 213 & 0.0004432 & 1.91 & 213 & 0.0004253 & 2.334 & 213 & 0.0004233 & 2.971 \\
\hline 4 & 4 & 125 & 0.000379 & 3.708 & 295 & 0.0002559 & 9.887 & 157 & 0.0004863 & 6.608 \\
\hline 8 & 4 & 148 & 0.000283 & 15.519 & 130 & 0.0003292 & 14.527 & 166 & 0.0002661 & 22.532 \\
\hline \multirow[t]{3}{*}{12} & 4 & $* * *$ & $* * *$ & $* * *$ & 92 & 0.0003097 & 21.627 & 125 & 0.0003779 & 35.959 \\
\hline & & \multicolumn{3}{|c|}{$\mathrm{s}=1$ is standard Orthomin $(\mathrm{k})$} & \multicolumn{6}{|c|}{ eps $=1.0 \mathrm{E}-6 \quad \mathrm{~N}=130$} \\
\hline & & \multicolumn{5}{|c|}{ CPU time $=1$ processor CPU time in seconds } & $* * *=\mathrm{pr}$ & L & & \\
\hline
\end{tabular}

Table 3.2. p-Orthogonal s-Step Orthomin Compared to $s$-Step Orthomin for Ill-Conditioned Matrix (eps=1.0E-10).

\begin{tabular}{|c|c|c|c|c|c|c|c|}
\hline \multirow[b]{2}{*}{ s } & \multirow[b]{2}{*}{ k } & \multicolumn{2}{|c|}{ s-Step Orthomin } & \multicolumn{2}{|c|}{$\begin{array}{l}\text { p-Orthogonal s-Step Orthomin } \\
\text { Modified Gram-Schmidt }\end{array}$} & \multicolumn{2}{|c|}{$\begin{array}{c}\text { p-Orthogonal s-Step Orthomin } \\
\text { Householder }\end{array}$} \\
\hline & & iterations & max error & iterations & max error & iterations & $\max$ error \\
\hline 1 & 1 & $701^{*}$ & 18811 & - & - & - & - \\
\hline 2 & 1 & 44 & 0.0001447 & 44 & 0.0003902 & 44 & 0.00040967 \\
\hline 4 & 1 & 29 & 0.0006337 & 23 & 0.0001125 & 23 & 0.00009717 \\
\hline 8 & 1 & 21 & 0.00207 & 11 & 0.00008743 & 10 & 0.001257 \\
\hline 12 & 1 & 22 & 0.0175 & 7 & 0.000002226 & 7 & 0.00001121 \\
\hline 16 & 1 & 30 & 41.267 & 6 & 0.0003545 & 6 & 0.00002671 \\
\hline 20 & 1 & 45 & 471.2 & 5 & 0.0001649 & 6 & 0.00003692 \\
\hline 1 & 4 & $701^{*}$ & 14556 & - & - & - & - \\
\hline 2 & 4 & 38 & 0.0001134 & 128 & 0.0009316 & 138 & 0.001331 \\
\hline 4 & 4 & 37 & 0.0006885 & 84 & 0.0002993 & 87 & 0.000103 \\
\hline & & $\begin{array}{l}\text { NOTE: al } \\
*=\text { proble }\end{array}$ & $\begin{array}{l}.0 \mathrm{E}+6 \\
\text { ed iteration }\end{array}$ & $\begin{array}{l}s=1 \text { is } \operatorname{stan} \\
\text { limit }\end{array}$ & thomin $(k)$ & & \\
\hline
\end{tabular}


Table 4.1. Vector Performance of the $A^{T} A$-Orthogonal s-Step Code Using the PERFTRACE Performance Analysis Tool

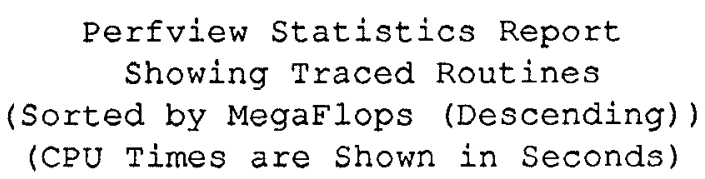

\begin{tabular}{|c|c|c|c|c|c|c|c|c|}
\hline Name & Called & Time & Avg Tim & EX \% & $A C M$ \% & Mmems & Mflops & \\
\hline-------- & ------ & $-----n$ & $------m$ & ----- & ----- & ---- & ---- & \\
\hline SORTHOMIN & 1 & $2.96 \mathrm{E}+00$ & $2.96 E+00$ & 59.9 & 59.9 & 344.3 & 249.7 & 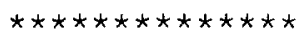 \\
\hline MATVEC & 706 & $4.52 E-01$ & $6.40 E-04$ & 9.1 & 69.0 & 290.6 & 237.7 & $\star \star$ \\
\hline MGS & 87 & 1. $50 \mathrm{E}+00$ & $1.73 E-02$ & 30.4 & 99.4 & 313.2 & 195.7 & $\star \star \star \star \star \star \star * *$ \\
\hline MESH & 1 & $2.44 E-02$ & $2.44 E-02$ & 0.5 & 99.9 & 48.9 & 165.4 & \\
\hline SMAIN & 1 & $3.58 \mathrm{E}-03$ & $3.58 E-03$ & 0.1 & 100.0 & 40.5 & 66.8 & \\
\hline \multicolumn{9}{|c|}{ 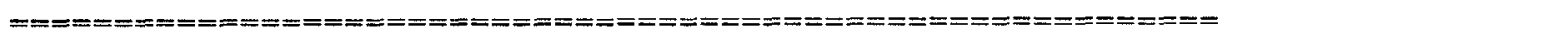 } \\
\hline otals & 796 & $4.94 \mathrm{E}+00$ & & 100.0 & 100.0 & 328.3 & 231.6 & \\
\hline
\end{tabular}

Table 4.2. Single Processor Performance.

\begin{tabular}{|l|ccc|cc|cc|cc|cc|}
\hline \multirow{2}{*}{$\mathrm{N}\left(\operatorname{size}=\mathrm{N}^{*} \mathrm{~N}\right)$} & \multicolumn{2}{|c|}{32} & 64 & 128 & \multicolumn{1}{|c|}{192} & \multicolumn{2}{|c|}{256} \\
\cline { 2 - 9 } & Time & Iter & Time & Iter & Time & Iter & Time & Iter & Time & Iter \\
\hline Orthomin(2) & 0.022 & 95 & 0.107 & 166 & 0.885 & 383 & 3.304 & 663 & 7.748 & 881 \\
2-step Orthomin(1) & 0.026 & 61 & 0.123 & 98 & 0.763 & 167 & 2.571 & 252 & 6.134 & 341 \\
$A^{T} A$-Orth. 2-step Orthomin(1) & 0.029 & 61 & 0.161 & 98 & 1.048 & 167 & 3.558 & 252 & 8.39 & 340 \\
$A^{T} A$-Orth. 8-step Orthomin(1) & 0.076 & 22 & 0.462 & 37 & 2.99 & 61 & 9.822 & 89 & 20.53 & 105 \\
p-Orth. 2-step Orthomin(1) & 0.037 & 61 & 0.188 & 98 & 1.205 & 168 & 3.992 & 251 & 9.634 & 342 \\
p-Orth. 8-step Orthomin(1) & 0.093 & 22 & 0.522 & 37 & 3.327 & 62 & 10.81 & 90 & 23.47 & 110 \\
& \multicolumn{7}{|c|}{ Time 1 processor CPU time in seconds } & & & & \\
\hline
\end{tabular}


Table 4.3. Parallel Computation Speed-up on a CRAY Y-MP8.

\begin{tabular}{|c|c|c|c|c|c|c|c|c|c|}
\hline & $\begin{array}{r}\text { Time } \\
1 \text { proc. }\end{array}$ & $\begin{array}{r}\text { Time } \\
2 \text { proc. }\end{array}$ & Speed-up & $\begin{array}{r}\text { Time } \\
4 \text { proc. }\end{array}$ & Speed-up & $\begin{array}{r}\text { Time } \\
6 \text { proc. }\end{array}$ & Speed-up & $\begin{array}{r}\text { Time } \\
8 \text { proc. }\end{array}$ & Speed-up \\
\hline 1-step Orthomin(2) & 7.95 & 8.83 & 0.90 & 8.36 & 0.95 & 8.21 & 0.97 & 8.88 & 0.90 \\
\hline 2-step Orthomin(1) & 6.28 & 4.87 & 1.29 & 5.79 & 1.08 & 4.92 & 1.28 & 5.16 & 1.22 \\
\hline 4-step Orthomin(1) & 8.78 & 6.35 & 1.38 & 5.19 & 1.69 & 5.45 & 1.61 & 5.02 & 1.75 \\
\hline$A^{T} A$-Orth. 2-step Orthomin(1) & 8.70 & 6.07 & 1.43 & 5.95 & 1.46 & - & - & 4.98 & 1.75 \\
\hline$A^{T} A$-Orth. 4-step Orthomin(1) & 12.30 & 7.24 & 1.70 & 5.37 & 2.29 & 5.07 & 2.42 & 4.56 & 2.69 \\
\hline \multirow[t]{2}{*}{$A^{T} A$-Orth. 8-step Orthomin(1) } & 21.69 & 11.75 & 1.85 & 7.46 & 2.91 & 7.19 & 3.02 & 5.76 & 3.76 \\
\hline & \multicolumn{8}{|c|}{$\begin{array}{l}\text { Time = elapsed time in seconds on a dedicated machine, } \mathrm{N}=256 \\
\text { 1-step Orthomin(2) is the standard Orthomin(2) algorithm }\end{array}$} & \\
\hline
\end{tabular}

Figure 4.1. Parallel Computation Speed-up on a CRAY Y-MP8.

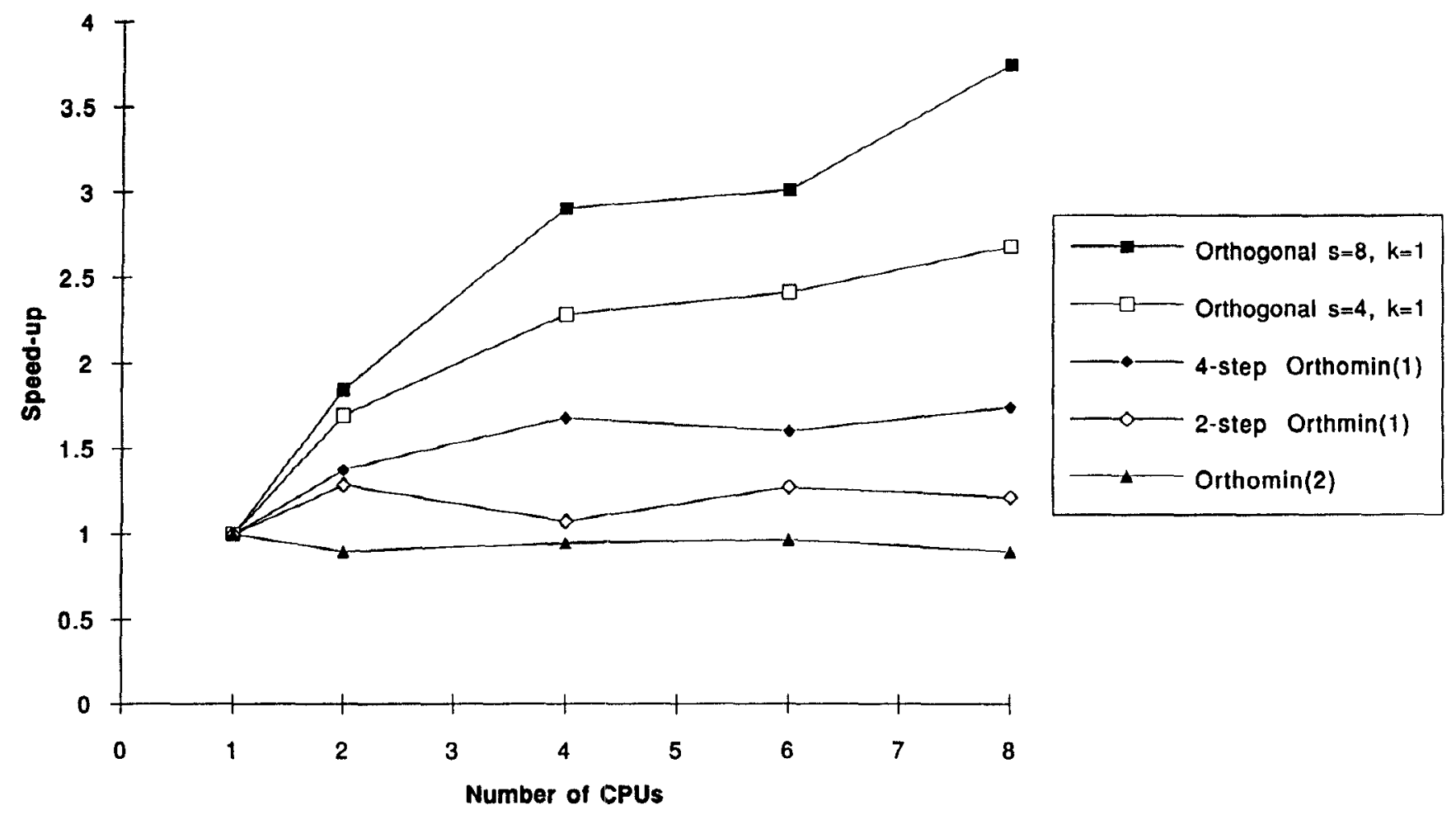

